\title{
Estudio sobre violencia intergeneracional
}

\author{
José Jesús Trujillo Vargas ${ }^{1}$ \\ Fecha: Recibido octubre 10 de 2014 - Aceptado noviembre 11 de 2014
}

\begin{abstract}
Resumen
La familia, como entidad educativo-social, se enfrenta a nuevos retos educativos diferentes a las exigencias de antaño. En la actualidad hemos pasado de un modelo patriarcal en donde todo se daba por supuesto y en donde existía una relación de dominio-sumisión de padres a hijos, a un modelo más igualitario, en contra de lo pensado, donde se están generando situaciones de violencia ascendente a nivel familiar, no por la filosofía misma del modelo, sino por la manera de gestionarlo, por la que están apostando los padres de hoy día. Así pues, este estudio intenta dilucidar alguna de las claves que están posibilitando esta situación familiar y social.
\end{abstract}

Palabras clave: familia, modelo, hijos, violencia ascendente

\section{Study of intergenerational violence}

\begin{abstract}
Family as a social-educational institution is currently facing new educational challenges different from requirements in the past. Nowadays we have moved from a patriarchal model where everything was taken for granted and where there was a control - submission relationship between parents and children to a more equal model which is raising violence situations in families. This is not due to the model philosophy but the way it is being managed by parents. So the aim of this study is to make clear some key issues for this familiar and social situation.
\end{abstract}

Keywords: family, model, children, upward violence.

${ }^{1}$ Psicopedagogo y doctor a través del Departamento de Psicología Social de la Universidad Pablo Olavide. Docente Investigador de la Corporación Universitaria del Caribe - CECAR. jose.trujillo@cecar.edu.co 
BÚSQUEDA - Julio / Diciembre de 2014 - No. 13 (41 - 59)

\section{Introducción}

El transcurrir de los tiempos afecta, de una u otra manera, a todas las instituciones educativosociales, las cuales tienen que ir redefiniendo su organización y funcionamiento en aras de satisfacer las nuevas necesidades que la sociedad va adquiriendo.

La familia, como institución educativo-social, no iba a ser menos, y en la actualidad intenta redefinir su papel a fin de que las relaciones convivenciales de sus miembros tengan un cariz de comprensión y confianza que ayude a mantener la armonía dentro de cada hogar.

Esto que suena tan armónico y propiciador del bienestar emocional de todos los miembros de cada ámbito familiar concreto, representa hoy día un reto importante para todos aquellos que se embarcan en el proceso de construir un ambiente familiar sano.

No siempre resulta fácil llegar a "buen puerto" en esta tarea, y por ello es necesario analizar e investigar las circunstancias que propician los desencuentros existentes entre padres o madres e hijos e hijas y las repercusiones que los mismos van teniendo a la hora de ir definiendo el papel de los más jóvenes en dicha institución y por ende, en la sociedad actual.

En determinadas ocasiones, el proceso de convivencia en el ámbito familiar recorre un camino no exento de "turbulencias", caracterizadas por faltas de respeto, agresiones verbales, falta de empatía..., entre padres e hijos/as que desembocan en frecuentes conflictos destructivos desde donde emanan procesos de violencia familiar.

Por tanto, desde nuestro campo de estudio y trabajo, que no es otro que el de la prevención e intervención en violencia familiar, desarrollado a través de la Asociación de Mujeres para la Formación y el Desarrollo (AMFORMAD)², esta investigación supone una oportunidad para evaluar una serie de aspectos claves, a tener en cuenta en las relaciones padres/madres e hijos/as:

- ¿Qué tiene que ver el establecimiento de límites, por parte de los padres hacia sus hijos/ as, con la educación en valores de los mismos?

- ¿Qué aspectos consideran los padres que hay que tener en cuenta a la hora de educar a sus hijos/as?

- ¿Qué grado de coherencia evidencian en la práctica como padres, en relación con lo que consideran fundamental para la educación de sus hijos?.

En primer lugar, se realiza un marco teórico para clarificar el concepto de violencia, indagando especialmente en la violencia intrafamiliar y se realizará un recorrido a través de diversas investigaciones en torno a la temática, para dar a conocer una cosmovisión más amplia del estado de la cuestión. Igualmente, se analizarán las claves para prevenir dicha violencia a través de la educación familiar.

En segundo lugar, presentamos una investigación que consta de varias partes. En una primera se recoge información de cuestionarios realizados por padres y madres, concernientes a aspectos que tienen que ver con la relación que guardan con sus hijos e hijas, e igualmente se recoge información de cuestionarios realizados a hijos e hijas relativos a las características que poseen las relaciones que estos mantienen con sus padres. En una segunda parte se recoge información de una serie de entrevistas semiestructuradas realizadas con la gran mayoría de los padres, madres e hijos/as que han realizado los cuestionarios de la primera parte. En la tercera parte se analiza información obtenida de las entrevistas individualizadas, realizadas en diferentes terapias en el centro de apoyo e intervención familiar donde trabaja uno de los siete equipos de trabajo que conforman la asociación AMFORMAD (Asociación de Mujeres

${ }^{2}$ Programa de Prevención e Intervención de la Violencia Familiar subvencionado por la Junta de Comunidades de Castilla la Mancha (España). 
para la Formación y el Desarrollo) subvencionada por la Junta de Comunidades de Castilla, la Mancha. En una cuarta parte se analiza la historia de vida de una mujer que acudió a nuestro servicio debido a las conductas negativas que su hijo presentaba en casa.

Tras la interpretación de los datos se realizan las conclusiones de la investigación y unas propuestas de acción futuras.

\section{Violencia familiar}

A continuación, se detallan una serie de aspectos concernientes a la violencia en general y a la violencia familiar en particular, que tratan de aminorar ciertos estereotipos que se pueda poseer sobre este tema en particular. Es por ello que desde un principio abogamos por clarificar el término violencia, diferenciándolo del de conflicto.

Se analizan algunas investigaciones que tratan de proporcionar una visión más amplia sobre la temática, a fin de posibilitar un conocimiento más específico y favorecer un estudio más pormenorizado que ayude a comprender el estado de la cuestión en la actualidad.

\section{Con qué está relacionada la violencia}

Desde hace unos años hasta nuestros días, se nos ha venido informando a través de los medios de comunicación de la existencia de casos de violencia de género, violencia hacia niños, hacia ancianos y más recientemente hacia adultos.

La violencia entre seres humanos tiene un origen bastante lejano en el tiempo y en parte, gracias a los sistemas políticos democráticos y a su consolidación; en la actualidad podemos hablar abiertamente sobre este tema y las personas objeto de violencia pueden denunciar su situación. Es por ello también, en parte, por lo que en muchos países africanos, asiáticos y latinoamericanos, la violencia es percibida como algo consustancial a las relaciones convivenciales, y su aceptación social hace que muchas personas que son maltratadas no se atrevan a intentar luchar por su derecho de no ser humilladas por cualquier otro semejante.
La violencia es un producto cultural. El ser humano, como cualquier otro animal, posee características biológicas que le inducen a la agresividad. Es por ello que todas las personas experimentan diversas emociones (miedo, agresividad, ira, tristeza,...) ante las diferentes situaciones que se les plantean en la vida cotidiana y estas emociones les llevan a "agredir" o enfrentarse con aquello que las produjo.

Las emociones desempeñan, así, una función adaptativa, proporcionando las conductas necesarias para la supervivencia de la persona en un momento determinado. Pero la agresividad puede pasar de ser un instinto útil, al servicio de nuestra supervivencia, a ser una conducta dañina para otro ser humano, por razones muy diferentes a la propia eficacia biológica. De modo que, es la cultura la que hipertrofia la agresividad convirtiéndola en violencia.

"La cultura juega, pues, un papel fundamental en la configuración del ser humano que, como cualquier otro animal, tiene una biología que le induce agresividad. Pero la cultura también puede hacer lo contrario e hipertrofiar la agresividad natural convirtiéndola en violencia" (Sanmartín, 2004, p. 21)

Así pues, todos somos agresivos por naturaleza, entendiendo la agresividad como un comportamiento instintivo de cara a la supervivencia (podemos actuar de forma agresiva con nosotros o con otros para salvar la vida), pero la violencia no es una conducta de supervivencia. Es selectiva (el agresor elige hacia quién dirige la violencia), es un ejercicio de poder. Por ello, dentro del hogar, el papel del agresor lo ejecuta quien tiene mayor poder (normalmente el hombre sobre las mujeres y los menores).

Por tanto, en cierto modo, "la violencia es muy humana, ya que está ligada al proceso evolutivo que ha conducido a la aparición del ser humano en la Tierra, y que no es tanto un proceso evolutivo natural cuanto una evolución cultural, artificial, que tiene al ser humano como sujeto agente y paciente a la vez" (Sanmartín, 2004, p. 23) 
En ocasiones, el hogar, la familia, las relaciones afectivas y todo lo que asociamos, en principio, a cariño, compañía mutua y satisfacción de necesidades básicas para las personas, pueden ser situaciones de riesgo para la aparición de conductas violentas.

Para ocultar dicha realidad existen una serie de mitos tales como que la violencia familiar es un fenómeno raro de escasa incidencia, que se produce sólo en familias muy desfavorecidas, que es la consecuencia de alguna enfermedad mental o que es la sociedad la que está enferma.

Otra de las creencias que intentan ocultar esta realidad es entender que este es un problema que pertenece al ámbito de lo privado, y que, por tanto, debemos tomar medidas sólo cuando se convierte en un delito.

Muchas formas de maltrato están respaldadas por normas sociales que perpetúan desigualdades y relaciones de poder abusivas dentro de las familias.

\section{Tipos de violencia y formas de ejecución de la misma}

Tradicionalmente se ha distinguido entre violencia física, psicológica o sexual, creemos que esta categorización puede llevar a confusión, ya que, por ejemplo, es difícil que exista una violencia que cause daños físicos y no psicológicos.

Asípues, desdenuestraasociación, diferenciamos los tipos de violencia en función del poder con que una o varias personas ejercen la violencia sobre otra u otras. Es por ello que diferenciamos entre: violencia agresión y violencia castigo:

\section{a) Violencia agresión}

Se da cuando dos o más personas poseen el mismo poder en la relación y tratan de resolver sus desavenencias a través de actos violentos. En este tipo de violencia cada uno de los miembros implicados presentaría (a nivel general) una buena autoestima, en tanto no solo reciben golpes e insultos, sino que también los llevan a cabo, es decir, a la par que son humillados, humillan a otros semejantes.

Suelen ser conscientes de que existe una relación turbulenta, pero generalmente resulta difícil hacerles comprender lo negativo de convivir de esta forma y mostrarles otros caminos alternativos para resolver sus conflictos. El ejemplo claro, que día a día nos encontramos en nuestros centros terapéuticos, es el de un padre o una madre que maltrata a su hijo o hija a la par de que son maltratados por éste o ésta.

\section{b) Violencia castigo}

Se da cuando una o más personas ejercen un ejercicio de poder sobre otra u otras y él mismo se traduce en un castigo físico y psicológico constante en el tiempo. En este tipo de violencia cada uno de los miembros implicados presentaría (a nivel general) una baja autoestima, en tanto en cuanto unos son los "recibidores de golpes" y otros son los que los ejecutan, por lo tanto no existe esa compensación físico-emocional como en el anterior tipo de violencia.

En este caso es difícil hacer comprender tanto al maltratador como a la víctima que existe una situación que hay que modificar por el bien de todos, ya que generalmente el maltratador ha conseguido a través del tiempo que la víctima acepte este rol.

El ejemplo clarividente de este tipo de violencia es el de una mujer maltratada por su marido en el ámbito familiar, aunque otros ejemplos serían: padres maltratados por sus hijos o hijos maltratados por sus padres.

Estévez (2005) recoge en su estudio otras clasificaciones recientes y complejas del comportamiento violento, que hacen una distinción doble y diferencian entre varias formas de violencia (por ejemplo directa, física o manifiesta versus indirecta, verbal o relacional) y entre varias funciones de la violencia (reactiva o defensiva versus ofensiva, proactiva o instrumental). 
Tabla 1. Tipos de Violencia

\begin{tabular}{|l|l|}
\hline \multicolumn{1}{|c|}{ VIOLENCIA AGRESIÓN } & \multicolumn{1}{c|}{ VIOLENCIA CASTIGO } \\
\hline Relación entre iguales & Relación desigual \\
Intercambio golpes & Malos tratos típicos (físicos, psicológicos, \\
Pausa & emocionales) \\
- culpa & Se justifica el castigo \\
- reparación & No hay pausa \\
Se pide perdón & Uno es superior y el otro lo acepta \\
Se reconcilian & Ambos suelen presentar baja autoestima \\
Ambos suelen tener buena autoestima & Pronóstico reservado \\
Pronóstico positivo & \\
\hline
\end{tabular}

Fuente: Adaptado de Perrone y Nanini (1998)

Tabla 2. Formas de Violencia

\section{FORMAS DE VIOLENCIA}

La violencia directa o manifiesta se refiere a comportamientos que implican una confrontación directa hacia otros con la intención de causar daño (empujar, pegar, amenazar, insultar...)

La violencia indirecta y relacional no implica una confrontación directa entre el agresor y la víctima (exclusión social, rechazo social, difusión de rumores...) y se define como aquel acto que se dirige a provocar daños en el círculo de amistades de otra persona o bien en su percepción de pertenencia a un grupo.

\section{FUNCIONES DE LA VIOLENCIA}

La violencia reactiva hace referencia a comportamientos que suponen una respuesta defensiva ante alguna provocación. Esta agresión suele relacionarse con problemas de impulsividad y autocontrol y con la existencia de un sesgo en la interpretación de las relaciones sociales que se basa en la tendencia a realizar atribuciones hostiles al comportamiento de los demás.

La agresión proactiva hace referencia a comportamientos que suponen una anticipación de beneficios, es deliberada y está controlada por refuerzos externos. Este tipo de agresión se ha relacionado con posteriores problemas de delincuencia, pero también con altos niveles de competencia social y habilidades de líder.

Fuente: Adaptado de Estévez López (2005)

\section{Violencia adolescente}

Al margen de centralizar este punto en una $u$ otra teoría concreta, adquiere importancia reseñar que hay factores que avivan la "llama de los capítulos violentos", entre jóvenes y entre éstos y los adultos. Entre ellos se pueden mencionar: el uso nocivo que desde la televisión, muy especialmente, y demás canales informativos se hace de las noticias existentes sobre violencia en el ámbito familiar, aulas escolares, o demás espacios públicos o privados donde ocurren actos violentos, ya que sacan a la luz aquellos datos que nos informan de que han existido capítulos de violencia que han desembocado en muertes, en grabaciones que luego se han ido pasando vía email, o a través de internet... sin embargo, en pocas ocasiones dan a conocer el día a día en los hogares o demás espacios donde existe violencia.

La creciente incidencia de los casos de violencia de los menores, en su mayoría adolescentes, contra sus padres, nos alerta contra los efectos perversos atribuibles a los cambios educativos y sociales propios de nuestro momento histórico. 
BÚSQUEDA - Julio / Diciembre de 2014 - No. 13 (41 - 59)

La familia, a pesar de los cambios sociales que la han modificado, continúa siendo el primer y más importante agente para el desarrollo del individuo y su integración a la sociedad. Esta acción socializadora y facilitadora de aprendizajes se establece a partir de las costumbres, modelos de conducta, estilos de vida, normas y pautas de crianza que los padres imponen y aplican en la unidad familiar.

Ideas, valores y pautas de conducta son transmitidos, de forma deliberada o involuntaria, por la familia, siendo ésta una de las fuentes de variabilidad más importante para la explicación y predicción del comportamiento del niño.

Estamos viviendo un momento socio-cultural en el que han existido y siguen existiendo muchos cambios con respecto al estilo de comportamiento y normas que deben regir el funcionamiento de una familia.

Estas normas se han hecho más laxas, hay mayor permisividad en cuanto a lo que se tolera a los niños. Por otra parte, la administración de recompensas y castigos se hace de manera inconsistente, dependiendo del momento, el cansancio de los padres, la presencia de los otros, etc...

Hay menos tiempo para dedicarlo a la educación de los hijos, y éstos están expuestos a gran cantidad de entornos diferentes (el escolar, el de iguales, actividades extraescolares, televisión, etc.) con exigencias muy concretas y a veces incompatibles con las normas y valores de la familia.

El resultado de todo esto conduce a que los menores crezcan en un sistema educativo en el que apenas existe el "no", lo cual genera ausencia de tolerancia a la frustración, es decir, los adolescentes no admiten la negación de los refuerzos que demandan, lo cual desemboca en un comportamiento agresivo para conseguirlos.

Las formas de expresión más frecuentes de esta violencia familiar ofrecida por el menor son: violencia verbal, robos a los padres, chantaje, fugas, agresiones físicas, destrozos en la casa, etc.

En el marco de estudio de este trabajo nos movemos específicamente en el terreno de la violencia filio-parental, que se define como "el conjunto de conductas reiteradas de agresiones físicas -golpes, empujones, arrojar objetos-, verbales -insultos repetidos, amenazas- o no verbales -gestos amenazadores, ruptura de objetos apreciados- dirigidas a los padres o adultos que ocupan su lugar" (Pereira, 2006, citado en Pereira \& Bertino, 2010, p. 96).

Además de las funciones que hemos destacado, debemos añadir, otro factores como: el intento de desafiar y negar la autoridad paterna, lograr la atención y el protagonismo frente a los hermanos, evitar castigos y sanciones, conseguir la admiración y seguimiento por parte de hermanos pequeños, con el consiguiente efecto de modelamiento.

\section{La convivencia entre padres e hijos adolescentes}

La socialización es el proceso a través del cual el ser humano se convierte en persona y corresponde el primer trabajo desempeñarlo a los padres.

La socialización se define como el proceso por el cual los individuos, en su interacción con otros, desarrollan las maneras de pensar, sentir y actuar que son esenciales para su participación eficaz en la sociedad.

Este proceso, sobre todo en el ámbito familiar, casi nunca está exento de conflictos debido a multitud de factores, como pueden ser: distintas maneras de situarse ante el mundo, reconstrucción a nivel experimental, por parte de los hijos e hijas, de lo que significa bueno y malo, en función de sus vivencias y de lo que sus padres argumentan para dicha clasificación...

La socialización aporta dos aspectos fundamentales para la vida humana:

1. Facilita las bases y estructuras actitudinales para la participación adecuada y eficaz en la sociedad en la que vive. 
2. Hace posible la sociedad, a través de la transmisión de valores y principios éticos de convivencia de generación en generación.

La cultura son las pautas, normas o guías que construye y dirige una sociedad y la hace característica, y se transmite a través de la socialización. Por tanto, la socialización es el proceso, mientras que la cultura son las normas, las pautas y los valores que se transmiten en ese proceso.

Para la transmisión de la cultura y para la socialización es necesario que el sujeto cuente con un ambiente adecuado, que esté en un contacto comunicativo, afectivo y normativo favorecedor del desarrollo de la socialización.

En la familia, es fundamental la conducta de apego que tiene una importante función en la supervivencia, porque asegura la proximidad y la protección de los padres a los hijos durante un período prolongado de tiempo en que la debilidad del nuevo ser requiere de la asistencia directa y continuada de los adultos. El papel fundamental de los padres consiste en asegurar la supervivencia de los hijos y también su integración sociocultural.

La familia constituye en sí misma un contexto sociocultural a través del cual llegan a los niños muchas de las actividades y elementos que son característicos de esa cultura, logrando así que la mente infantil se llene de contenidos, normas y reglas de convivencia que le permita desarrollarse como ser social. El tipo de familia, en la que nace y se cría un niño, afecta significativamente a las creencias, valores, expectativas, roles, comportamientos e interrelaciones que tendrá a lo largo de su vida.

Cuando las reglas no están claras y las consecuencias por el incumplimiento de las reglas no existen o simplemente son incoherentes o injustas, se comienzan a desatar una serie de problemas en la convivencia familiar entre hijos y padres, que suele hacerse más acuciante cuando los hijos pasan por la época de adolescencia.

Se ha pasado de un modelo familiar de dominio-sumisión, en el cual los padres eran los que mandaban y los hijos eran los que ejecutaban, a un modelo más democrático, en donde los hijos tienen más poder de decisión a la hora de elegir qué quieren hacer en cada ámbito de su vida.

Los hijos adolescentes en la búsqueda de su identidad personal (referido a quién soy y qué quiero), frecuentemente observan a los padres como un obstáculo para definir qué tipo de personas son, ya que son estos últimos quienes instan a los mismos a cumplir ciertas normas y asumir ciertas responsabilidades que muchos de ellos no están dispuestos a llevar a cabo, por multitud de razones tales como: creer que no tienen que dar explicaciones de nada de lo que hacen, no estar acostumbrados a cumplir normas ni asumir responsabilidades a lo largo de sus vidas, observar a los padres como personas que están anticuadas y que intentan "fastidiarlos" con reglas arcaicas...

Los padres de hoy, ante las nuevas demandas sociales, se ven perdidos, sin las habilidades necesarias para educar a sus hijos en función de las necesidades de estos y con base en unos valores humanitarios consolidados.

En muchos casos se ha confundido ser un padre o una madre progresista con no poner ningún tipo de límites a los hijos e hijas, lo cual ha dado la oportunidad a estos de obtener un poder en el ámbito familiar que, en la mayoría de los casos, no saben gestionar.

Todo ello, junto a muchos de los aspectos analizados hasta el momento, ha ido deparando en situaciones de violencia intrafamiliar de hijos a padres, que a su vez está propiciando una nueva corriente de intervención terapéutica en el campo de la psicología familiar y por ende, social.

\section{Violencia intrafamiliar de hijos a padres}

Desde hace unos cuantos años, se viene oyendo hablar de casos de maltrato a mujeres a manos de sus maridos, aún cuando los mismos han existido desde tiempos inmemoriales. Esto es un claro ejemplo de que el reconocimiento de los derechos hacia las mujeres en el mundo desarrollado está "caminando sobre suelo firme". Por ello cada vez existen más estudios acerca de 
la temática y se escriben gran cantidad de libros y artículos en aras de dar cobertura educativoformativo-psicológica tanto a las mujeres objeto de maltrato, como a los profesionales que se dedican a la intervención con víctimas y maltratadores.

Sin embargo no debemos caer en la tentación de referirnos a la violencia familiar sólo para dar a conocer aspectos de la violencia de género, o más recientemente llamada machista. Existen otros tipos de violencia en el ámbito familiar como pueden ser: violencia entre hermanos, violencia de padres a hijos (frecuentemente silenciada por parte de los adultos debido entre otras cosas a que para que la misma salga a la luz depende de la gestión de personas adultas, e igualmente en muchos casos difícilmente reconocible debido a que, como en todos los tipos de violencia, el maltratador no reconoce su maltrato...), violencia de hijos a padres (hacia la que está orientada este estudio), violencia hacia los ancianos...

Así pues, por violencia intrafamiliar nos referimos a todas las situaciones o formas de abuso de poder o maltrato (físico o psicológico) de uno o más miembros de la familia sobre otro u otros miembros familiares. Dichos actos de maltrato se desarrollan en el contexto de las relaciones familiares y ocasionan diversos niveles de daño a las víctimas de esos abusos.

La violencia intrafamiliar siempre representa una forma de ejercicio de poder mediante el empleo de la fuerza, ya sea física, psicológica o económica, e implica la existencia de "un arriba y un abajo" reales o simbólicos, que adoptan habitualmente la forma de roles complementarios "padre-hijo", "hombre-mujer", "joven-viejo", entre otros. El empleo de la fuerza se constituye así en un método posible para la resolución de conflictos interpersonales, como un intento de doblegar la voluntad del otro, de anularlo precisamente en su calidad de otro. La violencia implica una búsqueda de eliminar los obstáculos que se oponen al propio ejercicio del poder mediante el control de la relación obtenida a través del uso de la fuerza (Corsi, 1995).

\section{Investigaciones recientes sobre violencia adolescente}

Este apartado trata de recoger conclusiones de algunos estudios que casan con lo que en este se viene estudiando para analizar el estado de la cuestión. Estas investigaciones nos dan a conocer una serie de conclusiones que de alguna manera explican la convivencia existente entre los hijos adolescentes y sus padres en el entorno familiar.

\section{Estudio $n^{\circ} 1$}

Pueyo en su estudio "Violencia juvenil, realidad actual y factores psicológicos implicados" (2001), en donde explica la extensión de este fenómeno y las posibles razones que justifican esta realidad, centrándose en las causas individuales y psicológicas que se encuentran detrás de la violencia juvenil.

Los objetivos que se proponen en este estudio son:

- Conocer la extensión del fenómeno de la violencia juvenil.

- Significar las razones básicas que justifican la realidad de la violencia juvenil, centrándose en las causas individuales y psicológicas que se encuentran detrás de este fenómeno.

En este trabajo se analizan los factores individuales que están relacionados con la llamada "violencia juvenil", la violencia que producen y sufren los individuos que en nuestras sociedades occidentales se sitúan en el rango de edad que va desde los 10 a los 29 años.

Durante el estudio, aunque no define claramente las variables de este, da a conocer que el mismo versa sobre la violencia juvenil, los problemas que los jóvenes presentan a nivel familiar y social, los factores psicológicos que subyacen ante dichos problemas y la posibilidad de que dichos actos violentos se transformen en actos delictivos en un futuro.

Las conclusiones que se desprenden del estudio son:

- Comprender la violencia juvenil requiere pensar en el joven como agresor y como víctima, 
ya que en ambos papeles encontramos a los jóvenes de forma muy frecuente.

- Analizar la importancia social de la violencia juvenil, para conocer su estado real, su dinámica y la prospectiva de la misma, requiere distinguir y tratar de forma distinta cada tipo de violencia que ejercen/sufren los jóvenes.

- Conocemos múltiples factores y razones aisladas que explican los porqués de los comportamientos violentos de los jóvenes, pero falta integrarlos en un marco general que permita proponer medidas eficaces de prevención y control de la misma.

\section{Estudio $\mathrm{n}^{\circ} \mathbf{2}$}

Álvarez Ruiz y Egea Marcos (2003), en su estudio "Aspectos psicológicos de la violencia en la adolescencia", realizan un repaso sobre las diferentes teorías de la agresividad y dan a conocer claves para la prevención e intervención en violencia.

El trabajo incide fundamentalmente en los aspectos psicológicos de la violencia. Tras un breve repaso por las diferentes teorías de la agresividad, los autores destacan la importancia de las relaciones tempranas; la enorme plasticidad del infante humano hace de esta etapa un momento crucial para el desarrollo evolutivo. La adolescencia va a definir la identidad personal de cada uno, por lo tanto, dichos autores tratan de definir en vez de clasificar al adolescente con conductas violentas. Por último dan a conocer aspectos a tener en cuenta para la prevención e intervención en violencia. Para todo ello delimitan su artículo en varios puntos:

\section{Teorías de la agresividad}

2. La sociobiología

3. El adolescente y la violencia

4. Características clínicas del adolescente violento

5. Conclusiones

Dentro de este último punto las más relevantes son:

- Cuando el niño siente que él es el dueño de la casa, o bien uno de los padres forma un tándem contra el otro, o incluso uno de los padres espera que culmine todas sus ambiciones, entonces es cuando el niño es fijado a un potente patrón de conducta en la que, el adulto va tener que ser necesariamente el preferido y el mejor. Su propio status va a ser lo único importante, y cualquier persona va a ser tenida o como un rival al que odiar o como un instrumento para su propio prestigio.

- De la misma manera, el niño que es rechazado y por lo tanto no suficientemente valorado, va a acarrear a lo largo de su vida un sentimiento de inferioridad, así como un daño a su propia autoestima. Este intenso sentimiento va a ser una fuente inagotable de ira y de odio.

\section{Estudio $\mathbf{n}^{\circ} \mathbf{3}$}

Estévez López en su tesis doctoral sobre "Violencia, victimización y rechazo escolar en la adolescencia" (2005), donde intenta conocer más en detalle algunos aspectos relacionados con el mundo adolescente y la vida de este en el contexto escolar.

Las variables principales consideradas en los distintos estudios que componen este trabajo pueden agruparse en: (1) variables escolares: violencia escolar, victimización, rechazo escolar, relación profesor-alumno, autoestima escolar y actitud hacia la escuela; y (2) variables familiares: comunicación con el padre, comunicación con la madre, apoyo parental, autoestima familiar y conflicto entre los cónyuges (agresión).

El objetivo general de esta tesis doctoral es analizar la relación existente entre determinadas variables individuales, familiares y escolares y los siguientes problemas de ajuste conductual y social en la escuela: (1) problemas de comportamiento agresivo/violento, (2) problemas de victimización por los iguales, y (3) problemas de rechazo escolar por los compañeros.

Entre las conclusiones más relevantes del estudio tenemos:

- Existe importancia de las figuras paterna y materna en los problemas de conducta en los hijos, de tal modo que, el comportamiento agresivo en la adolescencia no implica necesariamente la existencia de otros problemas de ajuste en el adolescente agresivo, como por ejemplo la 
BÚSQUEDA - Julio / Diciembre de 2014 - No. 13 (41 - 59)

aparición de síntomas depresivos, a menos que su comportamiento influya negativamente en las relaciones familiares con los padres.

- Los adolescentes con problemas de comportamiento en la escuela presentan índices más bajos de autoestima familiar y escolar que aquellos sin problemas de conducta.

\section{Metodología}

A continuación se expone la investigación realizada durante este proyecto, a fin de contrastar el marco teórico, anteriormente descrito, con la práctica real y diaria de los centros terapéuticos de AMFORMAD.

Sólo de este modo podemos ofrecer las características de nuestro trabajo diario, establecer líneas de intervención futuras y exponer conclusiones que puedan resultar de ayuda en futuras investigaciones sobre la temática.

\section{Planteamiento inicial y objetivos de la investigación}

Este estudio se centra sobre todo en la relación entre padres e hijos/as cuando estos alcanzan o están inmersos en el período de adolescencia. A fin de desmantelar estereotipos y prejuicios en las relaciones padres e hijos/as adolescentes, es necesario realizar una aproximación a la vida diaria de un grupo de personas (ya sean padres o hijos/as) que conviven en una situación de frecuentes altibajos en sus relaciones.

Así pues, temas tan importantes en el ámbito familiar como los límites que se establecen de padres a hijos/as, la educación en valores que éstos mismos transmiten a sus hijos/as, las habilidades sociales que unos y otros poseen para fomentar una convivencia familiar, y por ende social, sana, deben ser tenidos en cuenta en este estudio en aras de clarificar los posibles déficit educativos y a partir de ahí dar a conocer pautas que fomenten la reconstrucción de la convivencia entre unos y otros.

El objetivo general de este estudio es investigar sobre los posibles factores que determinan que las relaciones entre hijos y padres adquieran tintes violentos y las posibles líneas donde incidir, a nivel familiar, para reestructurar dichas relaciones.

Este objetivo general nos lleva a secuenciar una serie de objetivos específicos, que de alguna manera ayuden a orientar el estudio al que se hacía alusión:

- Averiguar los métodos de transmisión de valores de las distintas familias y cómo estos se hacen explícitos en el día a día de los adolescentes.

- Indagar sobre el estilo educativo de establecimiento de límites de cada familia y exponer a las mismas en qué puede repercutir esto sobre la actitud de sus hijos e hijas.

- Describir los aspectos del clima familiar y analizar su influencia en el día a día familiar.

- Conocer los antecedentes familiares de los miembros de cada familia para, a partir de ahí, indagar sobre los diferentes estilos educativos y la relación de estos con los de sus antecesores y sobre las conductas de cada miembro en particular.

- Evaluar las contestaciones de los cuestionarios y de las entrevistas semiestructuradas y comparar las mismas, con las contestaciones dadas por los usuarios a lo largo de las terapias individualizadas.

\section{Diseño de la investigación}

En la medida en que iba "tomando cuerpo" este proyecto, surgían cuestiones en torno a la manera de plantear la investigación y qué instrumentos eran los más pertinentes teniendo en cuenta nuestra experiencia sobre la temática, la disponibilidad de acceso a los informantes, la cantidad de tiempo que llevamos interactuando con los mismos, etc.

Como no solo nos interesa qué tipo de estilo tiene cada familia para el establecimiento y respeto de normas y límites; los valores que imperan en cada modelo educativo familiar; las habilidades sociales que poseen los distintos miembros familiares, y muy especialmente los adolescentes para desenvolverse en el ámbito 
familiar, sino, que además de todo eso, nos resulta de gran interés saber los antecedentes familiares, las sensaciones que los informantes experimentan en cada una de las interacciones con sus familiares, las emociones impregnadas en cada situación en particular, qué tipo de comunicación (o incomunicación) existe y en qué depara la misma, traducido al día a día de cada una de las familias; cómo se ha ido desembocando (en los casos que así sea) en una situación insostenible en las relaciones familiares; qué problemas familiares existen, además de los puramente convivenciales, y cómo los mismos determinan las relaciones entre cada uno de los miembros de cada familia..., hemos optado por llevar a cabo una investigación con claro tinte cualitativo, aunque no por ello se desechan otros métodos cuantitativos que aportan importantes datos relacionados con todo lo comentado.

Salamanca Castro y Crespo Blanco (2007), comentan que entre las características del diseño de investigación cualitativa se puede destacar:

- Es flexible y elástico, es decir, puede adaptarse a lo que se descubre mientras se recogen los datos, como ya hemos apuntado anteriormente.

- Implica la fusión de diferentes metodologías.

- Tiende a ser holista, ya que se esfuerza por comprender la totalidad del fenómeno de interés.

- Se concentra en comprender el fenómeno o el entorno social. No busca hacer predicciones sobre dicho entorno o fenómeno.

- Exige gran dedicación por parte del investigador, quegeneralmente deberá permanecer en el campo durante periodos prolongados.

- El propio investigador es el instrumento de investigación.

- Requiere de un análisis continuo de los datos, lo que determinará las estrategias a seguir.

- Impulsa al investigador a construir un modelo de lo que se intuye en el ambiente social o de lo que trata el fenómeno de interés.
- Analiza el cometido del investigador y sus propios sesgos o prejuicios.

\section{Paradigma y posicionamiento metodológico}

La investigación es un proceso riguroso, cuidadoso y sistematizado en el que se busca resolver problemas, intentando organizar y garantizar la producción de conocimiento o de alternativas de solución viables. No solo debe tratarse de un proceso rico para el investigador sino, que a la par, debe ser interesante para las personas investigadas y para todas aquellas personas que accedan a dicha investigación.

A fin de valorar muchas de las opiniones que padres e hijos tienen sobre el proceso de convivencia entre ellos, esta investigación apuesta por una metodología abierta y flexible, en la cual el estudio etnográfico representa una gran oportunidad para la consecución de los objetivos anteriormente expuestos.

Los estudios etnográficos describen las pautas culturales y de comportamiento, tal como son percibidas por el grupo investigado, utilizan estrategias para obtener y analizar datos de tipo subjetivo (Erickson, 1977 citado en Goetz y LeCompte, 1988).

Si la etnografía asume que las acciones sociales se producen en los participantes según su forma de percibir, entender, interpretar, juzgar y organizar el mundo, para investigar sobre las acciones sociales no hay una vía más propicia que la de preguntar directamente a los participantes por qué actúan de determinada manera. Esto quiere decir que el etnógrafo escudriña por qué las personas actúan tal como lo hacen y a qué obedecen sus acciones. En la etnografía, entonces, se asume la relevancia del papel del actor y se insiste en comprender las acciones sociales desde la perspectiva del propio actor.

Así pues, para hacer etnografía es necesario adentrarse en el grupo, aprender su lenguaje y costumbres, a fin de buscar adecuadas interpretaciones de los sucesos, si se tienen en cuenta sus significados; no se trata de hacer una fotografía con los detalles externos, hay que 
BÚSQUEDA - Julio / Diciembre de 2014 - No. 13 (41 - 59)

ir más allá y analizar los puntos de vista de los sujetos y las condiciones histórico-sociales en que se dan.

El acercamiento a la realidad convivencial de cada familia en particular es vital a la hora de analizar los datos recogidos con cada persona objeto de estudio, ya que no se trata de que los resultados que se obtengan se puedan generalizar a otros ámbitos y otras familias, sino que se trataría de estudiar las posibles causas de la violencia existente en el seno de la sociedad y sentar bases para establecer una posible línea de intervención en programas educativos al efecto. Todo ello no nos exime que existan características y déficits comunes en las relaciones existentes entre las familias estudiadas y otras que aún no han sido objeto de estudio.

El análisis de datos etnográficos se verifica a través de un diálogo permanente entre los científicos y el grupo social en estudio, con el fin de negociar los significados y constituir realidades comunes. Implica la máxima coherencia posible entre los distintos momentos del proceso, para garantizar que no haya un paso en falso, que cada resultado proviene de un momento anterior visible por todos.

Por otra parte, exige una revisión de la relación teoría-método, en el sentido de lograr que la teoría explique esos datos específicos, y que esos datos específicos sirvan para revisar (y modificar) la teoría.

Todo lo argumentado no es indicativo de que en este estudio excluyamos técnicas de investigación de mayor carácter cuantitativo como pueden ser los cuestionarios, ya que los mismos nos aproximan a la realidad de las familias de otros centros donde intervienen profesionales del programa al que pertenecemos.

En el análisis del centro donde intervenimos, apostamos por ir comparando las respuestas obtenidas en los cuestionarios con las entrevistas individualizadas que mantenemos con los usuarios objeto de estudio.

\section{Variables de estudio}

El estudio presenta las siguientes variables:

- Variables generales: relación entre padres e hijos y violencia de hijos hacia padres. Estas variables representan la base de este estudio y aparecen reflejadas, de manera indirecta o directa, en todos y cada uno de los apartados que componen el mismo.

- Variables específicas: establecimiento de límites de padres a hijos, educación en valores y habilidades sociales de unos y otros. Las mismas influyen directamente en las generales, de tal modo que sin un coherente establecimiento de límites, sin una verdadera educación en valores y sin un correcto entrenamiento en habilidades sociales, existen riesgos mayores de que las relaciones entre padres e hijos se deterioren hasta tal punto de desembocar en violencia.

\section{Muestra}

La muestra de esta investigación está compuesta por un total de 49 familias de diferentes poblaciones de Castilla la Mancha. De esas familias, la investigación se ha realizado con 75 personas; 38 son adolescentes o preadolescentes, y 37 son padres o madres (10 y 27, respectivamente).

Los adolescentes tienen edades comprendidas entre los 10 y los 18 años, con una media de edad de 14,4 años, mientras los padres tienen edades comprendidas entre los 29 y 59 años, con una media de edad de 43,2 años.

La selección de la muestra se realizó a través de un procedimiento probabilístico sistemático, en torno a los usuarios que acuden a nuestro servicio, con lo cual existían familias que llevaban un mes de intervención y familias con mucho mayor bagaje dentro de nuestro servicio.

\section{Instrumentos para la recogida de datos}

A continuación se detallan los instrumentos utilizados durante este estudio, que distribuimos a través de varias partes:

$1^{\text {a }}$ Parte: Donde se elabora un cuestionario de 30 preguntas cerradas, con cuatro posibles 
respuestas, destinadas tanto a padres y madres por un lado, y un cuestionario de 20 preguntas destinadas a hijos/as adolescentes por otro. Dicho cuestionario ha sido pasado a 75 sujetos (38 adolescentes y 37 padres/madres).

En el cuestionario dedicado a padres y madres indagamos en cuestiones relativas a:

- Qué son los límites, para qué sirven, cuándo se establecen en casa, si tienen en cuenta las edades de sus hijos e hijas y si sirven para realizar la tarea encomendada, si son los mismos límites que sus padres utilizaban...

- Qué tipo de consecuencias utilizan con sus hijos e hijas cuando estos se saltan los límites.

- Qué tipo de responsabilidades tienen los hijos e hijas en casa.

- Qué relación guardan entre los padres y si están de acuerdo a la hora de educar a sus hijos e hijas.

- Con qué tiene que ver la educación en valores, la empatía y la asertividad.

- Si han existido capítulos de golpeos en casa.

- Si se consideran que sus hijos e hijas están contentos por tenerlos como padre o madre. Y si se consideran justos con sus hijos e hijas.

En el cuestionario dedicado a hijos e hijas indagamos en cuestiones relativas a:

- Tipo de límites que tienen en casa.

- Tipo de relación que guardan con sus padres.

- Si respetan más a uno o a otro o a los dos por igual.

- Qué tipo de responsabilidades tienen en casa.

- Si les han golpeado o han golpeado a alguien alguna vez.

- Si son empáticos.

- Si se alteran mucho cuando algo no les sale bien. $2^{a}$ Parte: Consta de un cuestionario con preguntas abiertas para padres y otro cuestionario para hijos e hijas (consultar anexo I), las cuales pueden ser modificadas y reorientadas o no, en función de las contestaciones de las personas que las realizan. Dichas entrevistas han sido pasadas a 66 sujetos, quienes también han realizado los cuestionarios cerrados.

La entrevista semiestructurada para unos y otros gira en torno a:

- Reacciones de unos y otros ante las malas conductas de los chicos.

- Tipos de problemas existentes a nivel familiar.

- Tipo de relación entre padres e hijos.

- Significado de los valores.

- Cómo se resuelven los conflictos en casa.

Tanto los cuestionarios como las entrevistas semiestructuradas nos permiten acceder a un mayor grupo de personas a la hora de realizar la investigación y de esta manera evaluar el tema en cuestión de una manera más amplia, sin embargo, en los mismos, puede que los sujetos objeto de investigación contesten a las preguntas pensando en lo que deberían contestar, más que en lo que realmente está pasando en su ámbito familiar.

Es por ello que en el centro $n^{\circ} 7$ de AMFORMAD, donde intervenimos diariamente con familias, realizamos un análisis comparativo entre las contestaciones que los usuarios dan a conocer en los cuestionarios con aquellas que manifiestan en las entrevistas individualizadas.

$3^{a}$ Parte: Entrevistas individualizadas que realizamos con las familias usuarias de este servicio, que me da la oportunidad de paliar los posibles sesgos de los dos métodos anteriores, ya que son entrevistas realizadas semanalmente, en los que vamos conociendo las vicisitudes de la vida de dichos usuarios y nos da la oportunidad de analizar en qué grado son coherentes con las contestaciones realizadas en los cuestionarios anteriores. A parte nos da la posibilidad de conocer y dar a conocer aspectos personales de cada familia, de una manera cercana y 
BÚSQUEDA - Julio / Diciembre de 2014 - No. 13 (41 - 59)

aproximada a la historia de vida de cada una de ellas en particular.

Para estas entrevistas individualizadas hemos analizado la información obtenida del trabajo con 12 familias, muchos de los miembros de cada una han realizado el cuestionario y la entrevista semiestructurada.

4 Parte: Historia de vida de una mujer que ha sufrido malos tratos en su ámbito familiar por parte de su hijo menor, el cual actualmente y tras varias denuncias por parte de su madre, está cumpliendo una medida judicial en un centro de menores.

\section{Procedimiento de análisis de datos}

Los participantes de esta investigación eran escogidos a través de un muestreo probabilístico sistemático, de tal modo que se escogieron una serie de días en cada uno de los centros y un número de sesión, que iba cambiando de un día a otro, en donde se iban a pasar los cuestionarios. A la persona con quien teníamos que intervenir ese día y a esa hora, se le pasaba el cuestionario y la entrevista semiestructurada y también se le podía pasar a algún miembro familiar, siempre y cuando tuviéramos una sesión no alejada en el tiempo con dicha persona o personas. En los quince últimos minutos de cada sesión, las personas objeto de estudio eran invitadas a realizar los cuestionarios y las entrevistas semiestructuradas; en el caso de que no les diera tiempo a responder a todas las cuestiones, podían llevarse el cuestionario a sus domicilios para poder finalizarlos. A cada uno de los investigados se les explicaba el cuestionario a realizar y se les pedía consentimiento para que los datos obtenidos del mismo, pudieran ser utilizados para la elaboración de este estudio.

\section{Interpretación de los resultados}

Los padres, y muy especialmente las madres, acuden a nuestro servicio con un grado de incertidumbre, y ansiedad, considerable. Se encuentran "encerrados" en un proceso "negativo" en el que no encuentran salidas; generalmente suelen pensar que lo han probado todo y que no hay nada que les pueda ayudar realmente y acuden a nuestro servicio como la última posibilidad para reestablecer la relación con sus hijos e hijas.
A medida que avanzamos en la intervención con ellos y vamos dando estrategias para un mayor acercamiento de estos hacia sus hijos e hijas, y vamos estableciendo pautas para que adquieran el "poder" en casa, el nivel de incertidumbre decae, en cierto modo, y comienzan en la mayoría de los casos a "hacer algo" de lo indicado para cambiar la situación existente en casa.

Es entonces, cuando, frecuentemente, comienzan a darse cuenta que las normas que establecen en casa han sido y siguen siendo poco coherentes, descoordinadas, con diferente intensidad, incluso ante las mismas conductas negativas por parte de sus hijos e hijas; a veces se muestran demasiado permisivos y otras veces intentan mostrarse directivos cuando intuyen que las situaciones les desbordan, que conceden excesivos privilegios a sus hijos e hijas e incluso cuando estos últimos no han sido merecedores de los mismos o no han demostrado que sean capaces de gestionar dichos privilegios...

Existe la posibilidad de que el proceso violento esté tan establecido en casa, que los padres no se vean con capacidad para cambiar ni un ápice en la relación con sus hijos e hijas; evidentemente en estos casos resulta más complicada la intervención y suele durar más tiempo. También depende, entre otros muchos factores, del carácter que posean los padres y cómo proyecten dicho carácter ante estos problemas.

Podríamos diferenciar entre varios tipos de padres:

- Aquellos que llevan a cabo todo lo trabajado durante las sesiones y que están deseando saber más cosas para aplicarlas en casa.

- Aquellos que llevan a cabo parte de lo trabajado en las sesiones y que no están totalmente convencidos de que esto pueda servir para algo.

- Aquellos que llevando o no a cabo, lo trabajado en las sesiones, piensan que su problema familiar, difícilmente se podrá subsanar. 
Así pues, los cuestionarios nos pueden dar a conocer posibles errores existentes en las concepciones educativas de los padres, aunque también, de alguna manera, en función de la intencionalidad de estos y de lo trabajado en sesiones, nos puede "ocultar" algunos problemas existentes en el modelo educativo de los mismos. Es decir, puede que hayan existido padres cuyas respuestas vaya en función de lo trabajado en sesiones, pero que en realidad no sea ni lo que ellos piensan, ni lo que llevan a cabo en el proceso educativo con sus hijos e hijas. Es por ello que en algunos casos, existen contradicciones entre lo que contestan en dichos cuestionarios y lo que comentan en las sesiones terapéuticas.

Cuando existen problemas de convivencia entre padres e hijos / as adolescentes, evidentemente esto indica la presencia de una crisis, en donde se está cuestionando por parte de estos últimos, el modelo educativo que sus padres han ido estableciendo a lo largo y ancho de sus convivencias. Sin embargo, los padres responsabilizan en la mayoría de las ocasiones a sus hijos e hijas y hacen afirmaciones tales como: "está en mi contra", "no me puede soportar", "antes me quería, pero ahora creo que no", "me quiere fastidiar la vida"..., son pruebas evidentes de que los hijos e hijas manejan la situación de casa a sus anchas y a través de la manipulación suelen conseguir beneficios de sus padres, en parte, porque estos se sienten culpables tras escuchar estos comentarios.

Los hijos e hijas suelen afrontar las situaciones familiares de forma distinta a sus padres, en tanto en cuanto son ellos quienes poseen gran parte de poder en casa y se resisten a que esta situación cambie, aunque en el fondo saben que alguien tiene que establecerles límites para su mayor bienestar emocional. Durante las sesiones, dan a conocer que no existen verdaderos problemas en las relaciones con sus padres, más allá de los existentes en otras familias.

Cuando los padres consiguen establecer algún tipo de límite en casa y poner alguna consecuencia a sus hijos e hijas, estos se sienten "desubicados", en gran medida, porque a lo largo de mucho tiempo no había existido ningún tipo de resistencia a sus conductas.

Los hijos y las hijas adolescentes se aferran a intentar conseguir sus objetivos, privilegios..., y para ello incluso, dan argumentos irracionales a sus padres para intentar convencerlos.

Cuando no consiguen lo que pretenden, en muchos casos desatan su ira contra sus padres, contra sus hermanos, contra el mobiliario de casa..., lo cual fomenta el desconcierto de muchos padres y madres, que acceden en muchas ocasiones a darles o dejarles hacer aquello que ellos piden, lo cual refuerza negativamente la actitud de los mismos y hace emanar en ellos el pensamiento de que sí ha sido posible conseguir sus propósitos de esta manera, en alguna ocasión, puede ser posible en futuras oportunidades.

Frecuentemente, debido a lo comentado y a otras razones como pudieran ser los prejuicios y estereotipos acerca de los psicólogos, los chicos y chicas adolescentes se muestran reticentes a acudir a nuestro servicio, y aluden que ellos no tienen problemas psicológicos, y que no están "locos". Cuando la situación en casa se torna un poco más "restrictiva", suelen acudir a dicho servicio y se "enganchan" en el mismo, cuando comprueban que los pensamientos que tenían a priori no se corresponden con la realidad.

Con todo lo explicado en la teoría, podemos tener una visión global de la problemática existente en las relaciones paterno-filiales; cuando nos introducimos en cada caso en particular, observamos que ante problemas parecidos, cada cual lo vivencia de una forma diferente en función de su carácter, personalidad, situaciones contextuales, otros problemas, antecedentes familiares..., es por ello que resulta tan compleja la intervención debido a que aunque la misma vaya progresando, siempre hay retrocesos y detrás de cada situación familiar existen personas que asocian unos determinados sentimientos a cada una de las mencionadas situaciones. 
BÚSQUEDA - Julio / Diciembre de 2014 - No. 13 (41 - 59)

\section{Discusión y conclusiones}

A lo largo de todo este estudio hemos ido descifrando los factores que determinan los procesos violentos que tienen lugar en la familia, en su versión intergeneracional (de hijos a padres). Tanto el marco teórico como la parte práctica de este estudio contienen aspectos que conviene resaltar, a fin de servir como posible ayuda a otros análisis relacionados con esta temática.

Durante el estudio, hemos ido analizando la violencia familiar y su desencadenamiento a través de falta de límites en la educación de los padres hacia sus hijos, las habilidades sociales que estos poseen y "transmiten" a sus hijos a la hora de socializarse con ellos y con otras personas, cómo incide todo ello a la hora de adquirir valores por parte de los hijos..., y todo lo anterior, fundamentado en la práctica diaria que llevamos a cabo en el servicio de apoyo familiar para el que trabajamos.

Para establecer estas conclusiones, se ha realizado un minucioso análisis de todo lo descrito en este estudio, así pues existen conclusiones relativas al marco teórico que sustentan y dan un valor añadido a las conclusiones que pertenecen a la parte práctica, unas sin las otras serían "insustanciales" y provocarían que dicho estudio fuera incompleto.

Las siguientes conclusiones no se proponen como un "recetario" a tener en cuenta a la hora de analizar la problemática existente en las relaciones hijos-padres, más bien suponen una oportunidad para ir descifrando claves en torno al por qué de la violencia intrafamiliar de los primeros hacia los segundos, teniendo en cuenta siempre la particularidad de que el estudio está realizado con familias asistentes a un servicio específico de intervención, existiendo seguramente muchos más casos familiares en donde ocurren sucesos violentos que no estén acudiendo a tratamiento psicoeducativo y de los que se desconoce su problemática específica.

Proponemos como conclusiones más reseñables de este estudio las siguientes:
- La violencia es un fenómeno propiciado y mantenido por factores de tipo cultural y social.

- La violencia familiar se asienta sobre premisas, valores y leyes muy arraigadas en nuestra cultura.

- Somos agresivos por naturaleza, entendiendo la agresividad como un comportamiento instintivo de cara a la supervivencia (podemos actuar de forma agresiva con nosotros o con otros para salvar la vida), pero la violencia no es una conducta de supervivencia. Es selectiva (el agresor elige hacia quién dirige la violencia), es un ejercicio de poder. Por ello, dentro del hogar, el papel del agresor lo ejecuta quien tiene mayor poder (normalmente el hombre sobre las mujeres y los menores).

- Los cambios en los modelos de convivencia familiar están incrementando otras formas de violencia doméstica como el maltrato a los padres, a los niños o a los ancianos.

- La familia, a pesar de los cambios sociales que la han modificado, continúa siendo el primer y más importante agente para el desarrollo del individuo y su integración a la sociedad. Esta acción socializadora y facilitadora de aprendizajes se establece a partir de las costumbres, modelos de conducta, estilos de vida, normas y pautas de crianza que los padres imponen y aplican en la unidad familiar.

- Los hijos adolescentes en la búsqueda de su identidad personal (referido a quién soy y qué quiero), frecuentemente observan a los padres como un obstáculo para definir qué tipo de personas son, ya que son estos últimos quienes instan a los mismos a cumplir ciertas normas y asumir ciertas responsabilidades que muchos de ellos no están dispuestos a llevar a cabo, por multitud de razones tales como: creer que no tienen que dar explicaciones de nada de lo que hacen, no estar acostumbrados a cumplir normas ni asumir responsabilidades a lo largo de sus vidas, observar a los padres como personas que están anticuadas y que intentan "fastidiarlos" con reglas arcaicas... 
- La violencia intrafamiliar siempre representa una forma de ejercicio del poder mediante el empleo de la fuerza, ya sea física, psicológica o económica, e implica la existencia de "un arriba y un abajo" reales o simbólicos, que adoptan habitualmente la forma de roles complementarios "padre-hijo", "hombre-mujer", "joven-viejo", entre otros. El empleo de la fuerza se constituye así en un método posible para la resolución de conflictos interpersonales, como un intento de doblegar la voluntad del otro, de anularlo, precisamente en su calidad de otro.

- Los niños/as necesitan comprender las normas que rigen su mundo y saber hasta dónde pueden llegar en su exploración constante cuando interaccionan con demás personas. Son los padres quienes en primera instancia deben afrontar y abordar esa labor a fin de potenciar que los mismos sepan diferenciar entre los actos que son familiar y socialmente aceptables de aquellos que no lo son.

- Si la educación es un instrumento valioso para la transformación humanizadora de la sociedad, no es precisamente porque permita la adquisición de conocimientos disciplinares, sino sobretodo porque auspicia formas de relacionarse unos con otros desde la generosidad inequívoca, desde la emoción y desde los sentimientos más profundos del ser humano. Encontrar el equilibrio entre esos dos tipos de conocimientos (disciplinar y experiencial o relacional), conocimientos por otro lado de diferente origen y naturaleza, constituye un motivador desafío para la educación.

- La adquisición de habilidades sociales por parte de los niños y adolescentes fomenta la adaptación de éstos al conjunto de la sociedad, para ello es crucial que haya una puesta en práctica diaria en el seno familiar de las mismas.

- Las consecuencias que los padres pueden establecer ante las malas conductas de sus hijos e hijas conviene que sean justas, y por otro lado, coherentes.

- Los hijos y las hijas suelen confundir intencionalmente o no, las obligaciones de sus padres, sobre todo de sus madres, con las responsabilidades que ellos y ellas debieran tener.

- Cuando acuden a nuestro servicio, los padres y las madres dan una visión del problema caracterizada por indicar que las dificultades que existen en el hogar son propiciadas por sus hijos e hijas, que son ellos verdaderamente quienes tienen los problemas y con quienes hay que intervenir.

- Muchos padres y madres, cuando acuden a nuestro servicio, indican que no saben qué hacer porque ya han probado todo tipo de castigos con sus hijos e hijas, y no funcionan.

- Como padres o educadores no debemos incurrir en un uso descontrolado e indiscriminado de autoridad sobre aquellos seres a educar, ya que de esta manera caeremos en un abuso de poder que puede traer consecuencias emocionales y afectivas negativas para los "receptores" de dicha educación.

- Los hijos e hijas se amoldan a los estilos educativos impuestos por sus padres y actúan en consecuencia, en la medida que los padres no se replanteen dichos estilos y actúen por inercia, correrán el riesgo de tener que amoldar el estilo educativo a las necesidades que sus hijos vayan presentando. Esto suele crear nociones en los padres tales como: no saber qué hacer, encontrarse perdidos, tener el pensamiento de que sus hijos quieren hacerles la vida imposible, pensar que la situación es demasiado negativa, que les supera y que ya no hay nada que se pueda hacer para mejorarla.

- Aunque pudiera parecer lo contrario, los hijos e hijas saben que sus padres les tienen que poner límites, aunque a veces no estén necesariamente de acuerdo con los que éstos últimos les establecen.

- En muchas familias, se tiene el pensamiento por parte de ambos progenitores, que el papel de autoridad debe ejercerlo el hombre en mayor medida que la mujer, e incluso las madres adoptan ese rol y a veces se ven impotentes de 
BÚSQUEDA - Julio / Diciembre de 2014 - No. 13 (41 - 59)

educar a sus hijos sin el apoyo de sus maridos. Sin embargo, en un gran número de casos con los que trabajamos, el papel de autoridad, por diferentes motivos, es realizado por las madres, con o sin ayuda de sus maridos o ex-maridos.

- Los padres utilizan unas pautas de transmisión (diálogo, ejemplo personal, cariño y ejercicio de la autoridad, motivación en el estudio, formación religiosa y moral) en unos porcentajes, en su mayoría, superiores a los porcentajes de los resultados de asimilación que, según estos, tienen los hijos. Los propios padres son conscientes de esta dicotomía entre su enseñanza y lo que asimilan sus hijos. Esta realidad los desanima y no saben cómo actuar.

- Los entrevistados, no tienen especialmente claro a qué se refiere el concepto de valores, y sus definiciones son poco precisas, recurriendo en muchos casos a ejemplos que de algún modo les ayudan a "salir del paso" a la hora de definir dicho concepto. En muchos casos se confunden los valores con las normas sociales.

- Los hijos e hijas suelen contestar que tendrá poco que ver la educación que ellos den a sus hijos con la educación que ellos han recibido. Muchos piensan que les darán a sus futuros hijos e hijas todo cuanto estos les pidan y que les dejarán muchísima más libertad.

- Los hijos e hijas suelen afrontar la situaciones familiares de forma distinta a sus padres, en tanto en cuanto, en muchos casos, son ellos quienes poseen gran parte de poder en casa y se resisten a que esta situación cambie, aunque en el fondo saben que alguien tiene que establecerles límites para su mayor bienestar emocional.

- Cuando los padres consiguen establecer algún tipo de límite en casa y poner alguna consecuencia a sus hijos e hijas, estos se sienten "desubicados", en muchos casos porque a lo largo de mucho tiempo no había existido ningún tipo de resistencia a sus conductas.

- Los hijos y las hijas adolescentes se aferran, en muchos casos, a intentar conseguir sus objetivos, y para ello incluso dan argumentos irracionales a sus padres para intentar convencerlos.

Para prevenir la violencia familiar, desde nuestro punto de vista, conviene dar a conocer una serie de pautas a los padres tales como:

a) Favorecer que nuestros hijos e hijas asuman responsabilidades, más allá de sus estudios.

b) Favorecer la autonomía de los mismos, que a la larga deparará en mayor independencia.

c) Establecer límites y normas claras e imponiendo consecuencias justas, cuando estos no se cumplan.

d) Fomentar habilidades sociales en los mismos.

e) Establecer una educación en valores, en la que la práctica de las acciones diarias de los padres sean consecuentes y coherentes con lo que se les pide que realicen a los hijos e hijas.

f) Enseñarles estrategias de resolución de problemas más allá de frases estereotipadas y carentes de valor humanitario, tales como: "si te pegan, pega".

g) Promover un diálogo activo con los hijos e hijas, más allá de meros reproches, que generalmente conducen a que los hijos e hijas dejen de contarles sucesos de sus vidas a sus padres.

h) Favorecer el control de los ataques de ira de los hijos e hijas. Más que razonar con ellos en momentos de frustración, hacerles ver cuando estén más calmados, cómo nos sentimos y explicarles lo improductivo de dichos ataques, a parte de establecer consecuencias a sus actos.

i) No entrar en luchas de poder con los hijos e hijas que no conducen a nada, más allá de pérdida de tiempo y ascenso en la crispación de los ánimos de una parte y otra.

j) Reconocer cuando nos equivocamos, para que a ellos les resulte más fácil hacer lo propio.

k) Respetar ciertas cosas que para ellos resultan relevantes como: estilo de vestir, a quién elige como amigos o amigas, música que escuchan..., al igual que necesitamos que ciertas cosas sean respetadas por los demás.

Los programas televisivos existentes, son un claro ejemplo de que existe un problema 
emergente y que hay que ir diseñando estrategias para atajarlo, en aras del bienestar y la salud emocional de las familias, y por ende, de la sociedad.

A través de este estudio, se pretende significar algunas de las cuestiones interesantes que determinan las relaciones entre padres e hijos, y cómo las mismas van cambiando a lo largo de la historia, ya que si antes, el poder y control en la organización familiar lo ejercían los padres, actualmente esto está cambiando, y es precisamente el por qué de ese cambio en el que nos hemos centrado para llevar a cabo dicha investigación.

\section{Referencias bibliográficas}

Álvarez Ruiz, A. \& Egea Marcos, F. (2003): Aspectos psicológicos de la violencia en la adolescencia. Estudios de Juventud, 6.

Corsi, J. (1995): Violencia familiar. Paidós, Buenos Aires.

Estévez López, E. (2005): Violencia, victimización y rechazo escolar en la adolescencia. Valencia: Servicios de Publicaciones de la Universidad de Valencia.
Goetz, J. P. \& LeCompte, M. D. (1988): Etnografía y diseño cualitativo en investigación educativa. Madrid: Morata.

Pereira, R. \& Bertino, L. (2010): Cuando los adolescentes toman el poder. Sistemas Familiares y otros sistemas humanos. Año 26, n¹, p.p. 93-115.

Perrone, R. \& Nannini, M. (1995): Violencia y abusos sexuales en la familia. Barcelona. Paidós.

Pueyo, A. (2001): Violencia juvenil, realidad actual y factores psicológicos implicados. Barcelona: Universidad de Barcelona.

Salamanca, A. B. \& Crespo, C. (2007): El diseño en la investigación cualitativa. Nure Investigación $n^{\circ} 26$.

Sanmartín, J. (2004): La violencia y sus claves. Barcelona: Ariel. 